\title{
OPEN
}

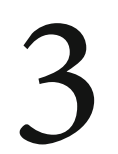

\section{Environmental Entanglements: Neurological Lives and Social Worlds}

\begin{abstract}
This chapter begins with the object that has perhaps most frequently underwritten collaborative work (including our own) between the social sciences and neurosciences: the effects of the environment. The chapter thus analyses a series of conceptual and methodological developments - in both biological and social domains - that have created the ground for life scientists and social scientists to begin collaborating around the simultaneously cultural and embodied effects of living in a particular environment. However, the chapter warns against some vitiated accounts of social life that can emerge from endeavours like these - and draws on recent research on the relationship between city life and mental health to begin thinking about how rich, thick and nuanced attention to the social can be traced through bioscientific methods.
\end{abstract}

Keywords: city; environment; interdisciplinarity; mental health; milieu; social neuroscience

Callard, Felicity and Des Fitzgerald. Rethinking Interdisciplinarity across the Social Sciences and Neurosciences. Basingstoke: Palgrave Macmillan, 2015. DOI: $10.1057 / 9781137407962.0006$. 


\section{Introduction}

In an article discussing the neural effects of the social environment, published in 2014 in the leading psychiatric journal Schizophrenia Bulletin, Lydia Krabbendam and colleagues pointed to the difficulty of investigating how city upbringing and minority status affect brain structure and function. In lamenting how 'the role of the social environment has been largely neglected in the search for the neural mechanisms underlying schizophrenia, they offered the following explanation:

For one thing, the complexity of social risks may have precluded researchers from trying to pin down the neural substrate underlying their effect. In addition, social risks have traditionally been the realm of social scientists and thus an improbable target for neuroscientists. (Krabbendam, Hooker, and Aleman 2014, 248)

Here, Krabbendam and her colleagues remind us of the legacy of a territorial division in which the neural has been handed over to the neuroscientists, and the social to the social scientists. This chapter comprises an effort to disrupt disciplines' careful tending of their own epistemological spaces, in order to figure out how those interested in understanding the tangled relations between the neural and the social - not least those whose research is devoted to understanding and intervening upon mental ill health and mental disorders - might work profitably together.

Many disciplines and epistemological domains now acknowledge that our neurobiology is intimately marked by the social, cultural, and environmental circumstances in which our lives take shape. Indeed, a range of disciplines and approaches - including, most prominently, social neuroscience (Cacioppo 2002), environmental epigenetics (see Niewöhner 2015; Pickersgill et al. 2013), and social epidemiology (Krieger 2001) - have lately emerged, or redefined their already-existing mission, in order to trace the multiplicity of ways in which, to use the cliché $d u$ jour, the social 'gets under the skin' (e.g. see Ferraro and Shippee 2009; Hertzman and Boyce 2010; Hyman 2009; McEwen 2012). Research foci range from interests in the role of socio-economic status in childhood neural development (Hertzman and Boyce, 2010), to the way that human social interaction becomes visible in brain function, to the role that the insults of poverty can play in the development of subsequent pathologies (Galea 2011). These approaches and areas, needless to say, have their own complex and contested genealogies, both internally and in relation 
to one another, as well as important differences in what exactly they intend by the 'social' or the 'environment' (or the 'social environment'). We will not attempt to disaggregate those differences here. What we do wish to stress is that a still-emerging, cross-disciplinary consensus on the developmental, and indeed ontological, inseparability of biological and social life underwrites many of the most compelling ways in which social scientists (and, at times, humanities scholars, when they can claim some purchase on investigations of the social and the cultural) and neuroscientists have learnt to labour together. Indeed, it is precisely the growing awareness of the importance of the environment (such is the favoured term) and of (social) experience in understanding both psychopathological and so-called 'normal' development that pushes the life and mind sciences to offer hospitality - if not to social scientists themselves, then at least to some of the concepts and phenomena with which they typically ply their trade.

Let us look briefly at how some of those invitations are crafted. Clinical psychologist Emily Holmes and colleagues, in calling for a strengthened 'mental health science' that will allow '[p]atients, mentalhealth-care providers and researchers of all stripes' to benefit, argue that: 'great strides can and must be made by focusing on concerns that are common to fields from psychology, psychiatry and pharmacology to genetics and molecular biology, neurology, neuroscience, cognitive and social sciences, computer science, and mathematics' (Holmes, Craske, and Graybiel 2014, 288). A recent Nature editorial, which endorsed the importance of interdisciplinary research involving the social sciences, cautioned that '[i]f social, economic and/or cultural factors are not included in the framing of the questions' posed by natural scientists working to 'deliver wonderful solutions to some of the challenges facing individuals and societies', then 'a great deal of creativity can be wasted' (Nature 2014, 5). Social psychiatrists Stefan Priebe, Tom Burns, and Tom Craig, proposing that 'the future of academic psychiatry may be social', have argued that a 'social paradigm ... would not ignore the neurobiological and psychological dimensions of mental disorders, but link them to social phenomena in the patient's life and in treatment' (Priebe, Burns, and Craig 2013, 320). Such an approach 'challenges the accepted distinction between basic and applied sciences' (whereby basic science discoveries are 'translated' into practice) by emphasizing that 'basic research on mental disorders as social phenomena would have to be conducted in the "real world"' (Priebe, Burns, and Craig 2013, 320). 
At the other end of the translational pipeline, Ralph Horwitz and colleagues warn that 'unless clinical, social, and environmental features that affect the outcomes of disease' are incorporated within genomicsbased knowledge, the current approach may be carving a path to " "depersonalized" medicine. On Horwitz's account, there has been the neglect of 'social and behavioral features that have been long disparaged as "soft" in measurement (because they often rely on subjective reports and physician assessment)' (Horwitz et al. 2013, 1155, 1156). The answer for Horwitz is to combat the atrophy of clinical science - by ensuring that physician investigators are not waylaid by 'quantitative models or reductionist science' so that they can focus on investigating the 'personal attributes of patients and their environments' (Horwitz et al. 2013, 1156). We are in agreement with Horwitz and colleagues. But one strand of argument that we wish to maintain in this chapter is that there has been no atrophy in the ranks of social scientists and humanities scholars: they - we - have long been working to understand the elements that go towards making up 'the complexity of human experience' that Horwitz is rightly convinced lies at the heart of understanding risks for disease as well as responses to treatment (Horwitz et al. 2013, 1156).

\section{The social in the neurosciences}

If 'the social' has never been as separate from the biological sciences as is sometimes now claimed, it remains true that there has been a qualitative shift, from the direction of the biological sciences, in perceptions of the grip that social life is thought to exert on the biology of the body. 'The recognition that most illnesses are the result of a joint contribution from both endogenous and exogenous factors', argue Satoshi Toyokawa and colleagues, implies that both psychiatric and non-psychiatric diseases are 'likely to have epigenetic etiologies that may reflect aspects of the social environment' (Toyokawa et al. 2012, 72). In a series of essays on what he terms a 'new social biology', the sociologist Maurizio Meloni roots this shift in a series of technical and conceptual developments within the biological sciences - from a renewed interested in altruism and forms of the 'prosocial' in evolutionary biology, to a realization of the force of social influence on the brain, to a consensus, in the wake of the completion of the Human Genome Project, that the genome is also 'reactive' to social and cultural influence (Meloni 2014b, 594). What such 
developments share, for Meloni, is that, in each, 'the traditional separation between the biological and the social has become increasingly difficult to define: biology has become porous to social and even cultural signals to an unprecedented extent' (Meloni 2014b, 594).

Let us zoom in on the relationship that especially interests us here that between the social and the neurological. The neuroscientists John Cacioppo and Gary Berntson, in their programmatic 1992 paper in American Psychologist, which proposed a 'multi-level analysis' of neurosocial interaction as their contribution to the then new American declaration of the 'Decade of the Brain', drew on the work of philosopher and psychologist William James to point out that 'the brain does not exist in isolation but rather is a fundamental but interacting component of a developing or ageing individual who is a mere actor in a larger theatre of life' (Cacioppo and Berntson 1992, 1020). For Cacioppo and Berntson, not only was this a realization that social processes are underwritten by neurological events, but that, in its turn, and crucially, social life folded back on the development and expression of individual neurobiology. They developed this perspective into a multiple and reciprocal determinism to insist that the promise of the Decade of the Brain would be realized only if we start to understand the ways in which 'the brain is a single, pivotal moment of an undeniably social species' (Cacioppo and Berntson 1992, 1027; see also Cacioppo et al. 2014 for specific reflections on psychiatry). There has not been a smooth passage from this generous proposal to an unproblematically reciprocal relationship between the neuroscientific and social sciences in the study of such events. It is worth noting, in this respect, that Cacioppo and colleagues, in a follow-up article in 2014 that described the potential contributions of social neuroscience to psychiatry, reported that:

To investigate the mutual influence of the biological and social environments and the mechanisms through which these influences operate, social neuroscientists, ranging from physicists to psychologists, epidemiologists to psychiatrists, philosophers to neurobiologists, and entomologists to zoologists, have begun to work together in interdisciplinary scientific teams using animal models, patient studies, and research on healthy individuals. (Cacioppo et al. 2014, 132)

That sociologists (or anthropologists, or geographers) are not picked out in this list is striking. It suggests the need for research to account for why interdisciplinary collaborations between neuroscientists and (particular kinds of) social scientists appear to have been harder to 
get off the ground than collaborations with, say, entomologists. We might nonetheless claim that Cacioppo and Berntson set the stage for a contemporary research landscape that - at least in certain corners - is attending carefully to the ways in which social adversity is a causal factor in mental disorder (European Network of National Networks studying Gene-Environment Interactions in Schizophrenia (EU-GEI) et al. 2014; Hyman 2009), in which the sociopolitics of stress and trauma are situated in accounts of specific psychopathologies (Galea 2011), in which specific brain regions are situated vis-à-vis the role they play in human social interaction (Amodio and Frith 2006), and so on.

From the other direction, a range of positions in social theory have radically undercut the traditional scepticism that the social sciences (at least the qualitative social sciences) have displayed toward not only biological explanations - but any admixture of the biological and social per se. In his account of what might become of 'The Human Sciences in a Biological Age', sociologist Nikolas Rose points out that, 'No longer are social theories thought progressive by virtue of their distance from the biological. Indeed the reverse assumption is common - it seems that 'constructivism' is passé, the linguistic turn has reached a dead end and a rhetoric of materiality is almost obligatory' (Rose 2013, 4). While retaining some critical distance from these obligations, Rose nonetheless casts a future for the social sciences in which an attention to vitality (understood not only as a philosophical commitment, but as a deep, abiding and collaborative attention to the biological stakes of human life) returns to the centre of the sociological enterprise. Similar claims have been made across a range of contemporary social theories (e.g. Braidotti 2010; Greco 2005; Grosz 2004). In our own efforts to think with and through bodies, we have been especially moved by arguments emerging from feminist science studies, and from that literature sometimes inadequately gathered together under the rubric of a 'material feminism' - which has, at least since Donna Haraway first drew attention to the theoretical potency of the body positioned scientifically (Haraway 1985), pioneered such approaches. Put very broadly, a 'material feminism' moves beyond an insistence on the construction of gendered bodies to ask whether feminist theory might, rather, seek resources in the materiality of the body, and in the thinking of the sciences that labour to bring that materiality into understanding. It describes a move of feminist theory beyond the 'textual, linguistic and discursive', to refocus on the pains and pleasures of embodiment: 'we need a way to talk about these 
bodies', Alaimo and Hekman point out, 'and the materiality they inhabit' (Alaimo and Hekman 2008, 3-4).

We have written elsewhere (Fitzgerald and Callard 2015) on the indebtedness of our own approach to Elizabeth Wilson's threading of neurology through feminist theory (Wilson 2004, 2011a, 2011b), and to Karen Barad's recasting of the relationship between political and physical worlds $(2007,2008,2011)$. Our immersion in these literatures has led us to challenge accounts and organizational structures that separate the practices and objects of the sciences (including the clinical sciences) from the practices and objects of social scientific and humanistic inquiries - accounts and structures that we have found to be common across the landscapes of interdisciplinary research focused on the mind and brain. We are quite convinced by Barad's account that 'even when the focus is restricted to the materiality of "human" bodies':

there are 'natural', not merely 'social,' forces that matter. Indeed, there is a host of material-discursive forces - including ones that get labeled 'social,' 'cultural,' 'psychic', 'economic,' 'natural,' 'physical,' 'biological,' 'geopolitical,' and 'geological' - that may be important to particular (entangled) processes of materialization. If we follow disciplinary habits of tracing disciplinarydefined causes through to the corresponding disciplinary-defined effects, we will miss all the crucial intra-actions among these forces that fly in the face of any specific set of disciplinary concerns. (Barad 2008, 128)

In short, if there is now an invitation to 'the social' from (some) parts of the biological sciences, then there is simultaneously an openness to think biologically from (some) parts of contemporary social theory. And if such moves are often partial and contested, they nonetheless form a gap into which the researcher interested in interdisciplinary experimentation might insert herself.

\section{Accounting for the social}

We are pitching for a grant about mind-wandering. It involves two social scientists (us) as well as a neuroscientist and a psychologist. At the heart of our bid are interdisciplinary experiments on mind-wandering, based on a workshop that will draw on accounts of distraction and daydreaming from the humanities and social sciences, as well as a reflexive ethnographic analysis of what that interdisciplinary process is actually like. Our collaborators have put together some excellent slides visualizing the state of the art in neuroscientific 
and psychological mind-wandering research. Des - struggling to illustrate what, exactly, an ethnographic analysis involves, and aware that he is handling this part of the pitch - talks to a slide featuring a photograph by Franz Boas of a man wearing a Kwakiutl whale-mask. We're all ready, after the presentation, to justify to the grant panel what, exactly, the hell this is doing there. But the questions from the panel, when they come, are almost exclusively about the imaging experiments we've proposed, and are directed squarely at our natural science collaborators, concerning the intricacies of our methods, the solidity of our conceptual underpinnings, and so on. The ethnographic portion of the project (which makes up the bulk of one of the two work-packages, i.e., more or less half the programme of research), is largely unremarked upon.

Afterwards, one of our collaborators wonders what it means when there is so much attention to what the experimenter will do, while the person whose presentation is centred on an image of a man in a whale-mask has something of an easier ride.

Our scientific collaborator diagnosed the situation, we believe, with great acuity. Here, we understand him to have been drawing our attention to the fact that while the funding panel was profoundly interested (and very well versed) in the nuances, specificities, and problematics of the novel experimental design that he proposed, there was no evidence of an equivalent focus on 'the social' or 'social relations', still less on the variable methods and attentions through which those phenomena, in their turn, might be brought to light. There was clearly room for the social to be brought into this space (and we do not diminish, on the part of the funding panel, the boldness and value of that move alone), and yet, beyond that generosity, there was still little scope to account for its specificities - or, indeed, to grapple critically with its own problematics, erasures, lacunae, and so on. The space was open to the social, certainly, but, for the two of us, at least, we had little sense that 'the social' itself might represent an important epistemological opening.

If an increasing attention to the social environment in the biological sciences can be read as an invitation to people in the social sciences, what 'the social' actually is in such spaces is often rather different from what it is within the social sciences themselves. This need not, of course, be a problem in itself: there are many models of the 'social', as well as of the 'neural', the 'cognitive', the 'affective', the 'cultural' (and so on) that course within and across a number of disciplines; such differences can themselves be revelatory to those exposed to them, and can precipitate new kinds of research questions as well as methodologies. What perturbs 
interpretive social scientists is when neuroscientific accounts and operationalizations of the social recapitulate ideas about culture and society that have long been repudiated - for epistemological, ontological, and political reasons - from many parts of the social sciences themselves.

Needless to say, there are analogous instances in which social scientists and humanities scholars employ outmoded or problematic or radically simplified concepts from the neurosciences - either to work with or to employ in severe critique of the science. We need think only of the way in which such concepts as 'mirror neurons' and 'plasticity' have been wielded outside of their home domains. Nonetheless, we well remember the interdisciplinary meeting in which the only other presentation, apart from our own, that attempted seriously to engage an account of social and cultural specificity did so by mobilizing the social-psychological bifurcation between 'individualist' and 'collectivist' cultures, which unproblematically split the Occidental from the Oriental (see Oyserman, Coon, and Kemmelmeier 2002 for a detailed critical review of this literature, from a psychological perspective). Certainly, some important notion of culture was at stake in this interdisciplinary proposal - and yet few sociologists, geographers, or anthropologists, and far from all psychologists and neuroscientists, would defend the notion of a 'Western' culture as a thing characterized by its members' individualism', whether by refusing to countenance that 'culture' is a thing (Mitchell 1995), by rejecting any isomorphism of space, place, and culture (Gupta and Ferguson 1992), or by critiquing any simple bifurcation between two kinds of people (individualist/collectivist) (Omi 2012). A number of other concepts that frequently surface in social and cultural neuroscience carry similar difficulties. Andreas Heinz and colleagues, for example, lament the "increasing use of the "race" concept in contemporary genetic, psychiatric, neuroscience as well as social studies' (Heinz et al. 2014, 1) - arguing that both this concept and, indeed, that of 'culture' (which they argue is often used as a proxy for 'race') carry within them the legacy of colonial attempts to categorize 'races' on the grounds of distinct physiognomies (see also the argument by the biologist William Klitz [2014]).

The point is that the roots of perspectives such as these on 'cultures' and 'races' lie not in accounts of society and culture as they have been opened up in disciplines such as sociology or cultural anthropology (at least in their more recent iterations), but rather in accounts of 'culture' that dominate the fields of cultural psychology and cultural neuroscience. In a well-cited review of cultural neuroscience, Joan Chiao, 
while tipping her hat to anthropologists such as Franz Boas and Margaret Mead, nonetheless figures what she calls 'culture-biology interactions' squarely within an account of 'culture' drawn from the narrower binaries that dominate cultural biology, viz. a literature that bifurcates 'cultures' along axes of 'individualism-collectivism, uncertainty avoidance, power distance, long-term/short-term orientation, and masculinity/femininity' (Chiao 2009, 288, 291; italics in original). Such binaries risk rendering static and inflexible apparent 'traits' that are seen to characterize particular groups of people ('cultures', 'races', 'genders') (see the critique by Martínez Mateo et al. 2012). What gets - or can get - neurologically accounted for, as aspects of the social environment, is here already radically constrained, and constrained precisely because it has not set foot outside the bounds of a particular kind of psychological thought. 'Psychology as a hub science', Chiao concludes her review, 'stands in a natural position to merge the scientific study of culture and biology by harnessing theories and methods from every area of psychology, from evolutionary and cognitive to cultural and developmental' (Chiao 2009, 300); such a perimeter seems to cut from consideration all those other disciplinary locations in which both culture and biology have been the focus of intense conceptual and empirical investigation.

As Svenja Matusall and her colleagues remind us, the specific form of 'the social' on offer in 'social neuroscience' can be rather a dispiriting one: 'currently, in neuroscience', they point out, 'the concept of "social" is a relatively static factor in experimentation... The approach towards studying the social via communal genetic make-up or individuals' brains is rather different from studying the external conditions for a social structure' (Matusall, Kaufmann, and Christen 2011, 14-15). In other words, there is a big difference between an experiment that differentiates (social) groups purely on account of their different physiological attributes, and one that explores how those differences might be produced through social as well as physiological patternings and dynamics. One might make, here, a lament - and others have done so - about the violence that fields crossing the neuroscientific with the social/environmental can sometimes inflict upon the hard-wrought claims of culture and society (e.g. Leys 2011). Indeed, one might well claim that not only is there not an especially enticing invitation to social scientists, from a neuroscientific field that imagines itself 'social' or 'cultural' - but that, worse, such fields, through powerfully reductive methodological and conceptual strategies, inflict significant damage on those very concepts. 
If we sometimes find ourselves in sympathy with such arguments, still we are loathe to wave yet another scrawny sociological fist in the general direction of the social neurosciences. What we have tried to do, instead, through the projects that we have been involved in, is to take seriously the question of which accounts of the social are on offer in the interdisciplinary neurosciences today (cf. Young 2011, 2012) - and to think, at the same time, about the value that might be placed on broader, livelier ways of working and thinking this notion. In this sense, we are trying to work through strategies through which more compelling accounts of the environment, of society, of culture, of milieu (to use the technical term of Goldstein 1995 [1934]) might yet get pulled through laboratorybased neurobiological studies. Because, as Svenja Matusall also reminds us, we can trace rather different accounts of the 'social' even within the relatively small world of social neuroscience - which is to say, this field is itself contested in various ways (Matusall 2013). The tension between the rather open account of the 'social' that appears in Cacioppo and Berntson, and the thinner, more vitiated descendant of that account that we read in much subsequent psychological work is sobering. The question, then, becomes: through which methods, strategies, and concepts, through which forms of alliance, and in relation to which research questions might these thicker and more capacious accounts of social life be mobilized and operationalized? To the extent that 'the social' or 'the cultural' is a way in to at least some forms of interdisciplinarity, how do you - whatever discipline you come from - get involved in projects where invigorating ways of conjuring one or both are centrally at stake?

\section{The urban brain}

One of the - many - uncomfortable facts about collaboration is that some kind of strategic reduction (of one's own concepts and immediate intellectual ambitions) is often the price of entry. One of our core messages is that learning to become an interdisciplinarian means coming to terms with that price. We would suggest, entirely seriously, and without prejudice, that any social scientist who is committed to writing 12,00o-word papers on different genealogies of 'the social', or who doesn't think she could bear the epistemic violence of using a construct like 'SES' (socioeconomic status) as a proxy for dense inter-lacings of class, social position, and education, is probably as well off not moving much beyond her 
sociology or anthropology department. By the same token, any neuroscientist who is unwilling to look beyond standard social-psychological literatures for accounts of 'culture' are equally advised to stay within (or accept that she is already staying within) the secure boundaries of a wellestablished, and singular, paradigm.

With that allowed, the mundane fact is that there are interdisciplinary projects where better and worse accounts and operationalizations of the social and of the cultural are on offer. One place to which we have turned for inspiration is the group of interdisciplinary researchers from the neurosciences and social sciences who are putting pressure on how sex and gender are conceptualized and operationalized in neuroscientific research (for a broad review, see Schmitz and Höppner 2014b). Anelis Kaiser and colleagues, for example, in a careful review of sex/gender differences that have been detected using fMRI, have demonstrated that focusing on sex/gender as a variable leads irrevocably to the detection of differences rather than similarities (Kaiser et al. 2009) - and have reflected on the epistemological and political implications of emphasizing such gender differences. Others have challenged core psychological constructs used within social and cultural neuroscience - see for example Daphna Joel and colleagues' study that challenged the category of 'core gender identity', which is frequently employed in neuroscientific studies (Joel et al. 2014). Or consider the work of those critical neuroscientists such as Suparna Choudhury, Lawrence Kirmayer, and Rebecca Seligman, who have mobilized the resources of anthropology and transcultural psychiatry alongside those of cognitive neuroscience to develop and operationalize less enervating accounts of the 'cultural' (Choudhury 2010; Seligman and Brown 2010; Seligman, Choudhury, and Kirmayer forthcoming; Seligman and Kirmayer 2008). Seligman and Brown, for example, have drawn on anthropological evidence to show that dissociative states are not only 'pseudo-adaptive' responses to stress, as they are frequently imagined in Euro-American psychiatric literatures, but can also be willingly inhabited, bringing to the fore 'forms of consciousness that allow individuals to enact alternative selves' (Seligman and Brown 2010, 134). This anthropologically influenced account of dissociation, moreover, can be used to parse neuroscientific data on the relationship between such dissociative states and inhibitory mechanisms, thus showing how anthropological evidence might be used to expand, torque and complicate neuropsychiatric data - without losing its specificity or force. 
We conclude this chapter by describing a project, whose contours we know particularly well, in which one relatively capacious account of the social is in play. The genesis of this project lay in three social scientists (Nikolas Rose, Ilina Singh, and DF) thinking through the long and tangled literature on the history of mental disorder in cities - focusing in particular on how the relations between the social and biological get recast in a range of different areas (see Fitzgerald, Rose, and Singh forthcoming, which provides a formal account of the thinking behind this project; cf. Singh 2012; Rose 2013). At the outset of this project, these three collaborators were each, in different ways, fascinated by a series of papers that had recently come out of the laboratory of the German neuroscientist and psychiatrist Andreas Meyer-Lindenberg - which purported to show, for the first time, and using brain-imaging methods, a biological mechanism through which the stress of urban life could be translated into a clinical pathology (Lederbogen et al. 2011; Meyer-Lindenberg and Tost 2012). City living has been associated with mental illness for at least a century: since the very emergence of psychiatric epidemiology (indeed, this is in some ways the founding insight of psychiatric epidemiology), we have known that mental illness associates with city life (Lewis and Booth 1994). And yet, despite a long and deep research literature, and despite the consistency of this finding, no one really knows why this is. There is no scholarly consensus around the classic debate between social causation (city living makes people mentally ill) and social drift (people who are already mentally ill tend to end up in cities for various reasons), while a long-standing literature on the 'social determinants' of urban mental illness has morphed into attention being given to the neurobiological and epigenetic mechanisms through which the tumult of city life gets into the skull.

Here, then, an interdisciplinary problem-space begins to emerge. Because not only is the relationship between urban space and mental health a well-known research area in epidemiology and social psychiatry: it also has a rich - but less storied - history in sociology too. Indeed, the question of the metropolis and mental life has classical antecedents in sociology (Simmel 2002 [1903]), while the question of 'mental disorders in urban areas' comprised one of the foundational research questions of American sociology (Faris and Dunham 1939). But what is more remarkable is this attention, and this link, to the biological and psychiatric sciences is, if not exactly forgotten, certainly not at the forefront of contemporary sociology. 
The 'urban brain lab' was developed at King's College London to work creatively with those heterogeneous and overlapping histories. Specifically, and precisely with a view to bring a simultaneously social and biological attention to the problem of urban mental health, the lab worked to assemble sociologists, neuroscientists, psychiatrists, epidemiologists, anthropologists, geographers, historians, and others around the question of urban mental illness - in order to develop novel conceptual and empirical projects. Distinct from much interdisciplinary research - which includes some, limited, social 'element' - the subterranean history of sociological attention to this question licensed the mobilization of sociological methods, histories, and concepts at the heart of the endeavour. Thus, at stake in this work is not the feeding in of sociological expertise into a neuroscientific project as such, but rather a bringing of the neurosciences into those questions - conceptual and empirical - that are preoccupying sociologists and those in proximate fields. What we would call the sociological social was, then, built into this project from the outset.

During 2014 and 2015, the lab ran two interdisciplinary events in London, which (a) drew together a range of psychiatric, biological, sociological, and historical attention to this question; but also (b) drove such attention, simultaneously, through the shared co-design of a novel empirical project for locating urban mental illness as a phenomenon that is lived on the streets as much as it is experienced in the body. The design of that project is, as we write, ongoing; but we hope that it may ultimately become a space for foregrounding at least one kind of sociological attention to the 'environment', and the pressure it exerts, in all its contest and complexity, through a nuanced and sophisticated attention to the embodiment of urban stress. It may show us, then, that even if we do sometimes encounter thin sociologies at the heart of avowedly interdisciplinary projects, there is no necessary relation between interdisciplinarity and vitiation. There are archives - historical and contemporary - that may yet help us to think and practise otherwise.

\section{Notes \& Queries: 3}

Q: A lot of my geographical and anthropological research has been preoccupied with socio-spatial configurations of different kinds of landscapes both urban and rural. How might this be of interest to interdisciplinary researchers addressing the mind and brain? 
Or:

Q: As a neuroscientist, I feel confident about how my field models genetic interactions, but it seems to me that accounts of social risk factors and life events are often much less robustly specified. Are there opportunities to work collaboratively across the disciplines on these questions?

A: In response to both of you, we emphasize that there are many compelling points of intersection of the neural and the social that might draw you into interdisciplinary collaborations. Here are a few of the many arenas that we think would benefit from greater interdisciplinary attention.

-Working up the complex intersections between environment, trauma, and mental ill health/psychosis. There is a growing body of evidence that links traumatic and other kinds of adverse experiences to the development of psychosis and other forms of mental illness (Varese et al. 2012). Researchers of all stripes know, however, that acquiring robust assessments of traumatic experiences is a very difficult task. There are many rich models of trauma in all its heterogeneity within the social sciences and humanities that attend to trauma's complex temporal structure, as well as the difficulties surrounding assessments of the veridicality of these experiences. How might these be threaded into clinical and epidemiological studies?

- Refining modelling of the environment in animal research. Many translational researchers have commented on the need for more ecologically valid models (particularly regarding the environment) in animal research (e.g. Nestler and Hyman 2010). Social scientists and historians of science and medicine have provided rigorous analyses of the assumptions and constraints built into models of the environment (Ankeny et al. 2014; Davies 2010; Ramsden 2011). How might these analyses be fed back into - and thereby refine - existing animal models?

- Improving models of stress as they entwine the physiological, the psychological, and the social. Neuroscientists know that there are multiple ways in which stress can affect brain function (e.g. Ganzel, Morris, and Wethington 2010). But while vulnerability factors have been extensively researched in relation to emotion, the relation between social behaviours and social brain function is far less well understood (Sandi and Haller 2015). In addition to large bodies of social scientific literature on how to conceptualize 
and study social behaviours, there has been significant historical and sociological research on the heterogeneous concept of stress as it moves between the physiological, psychological, and social (Cantor and Ramsden 2014; Jackson 2013). How might these different bodies of research be better brought together?

- Contributing to theorizations of - and operationalizations of 'human experience' that work simultaneously with neurological and socio-cultural data to develop new theoretical models and new experimental paradigms. One good example is the Hearing the Voice project - which focuses on the experience of hearing a voice in the absence of any speaker (often referred to as auditory hallucinations in psychiatry). Researchers have been bringing together phenomenological and other kinds of material from the humanities into neuroscientific and psychological models of voice hearing (e.g. Woods et al. 2014).

- Addictions. Anthropologists and other social scientists have argued for the importance of understanding the complexities of milieu and of trajectories of addiction (Raikhel 2015). Daniel Lende - one of the main proponents of the field of neuroanthropology (Lende and Downey 2012) - has shown how such a project becomes possible by bringing together biological measures and ethnographic data (Lende 2005).

- Modelling race, culture, gender, and sexuality. There are many ways in which the insights of the humanities and interpretive social sciences might bear on current thin (if not problematic) ways of categorizing individual and social identity in neuroscientific studies. We have already mentioned Joel and colleagues' work that pushes beyond existing psychological models of 'core gender identity' (Joel et al. 2014). Similarly, neuroscientific studies are beginning to add to and expand our accounts of how race operates and is mobilized as a social category (Kubota, Banaji, and Phelps 2012).

- Linking patterns of disorder or particular diagnoses to specific histories in particular areas. Sandro Galea's research in Detroit demonstrates how patterns of disorder or particular diagnoses can be traced to specific histories in particular areas (Galea 2011). How might historical sociologists and epidemiologists work together to develop other investigations into the entwinement of embodied stress and political histories? 
- Poverty. Research attempting to model the subjective insults incurred through poverty so as to understand how the effects of poverty 'get under the skin' often turn to ethological models (e.g. by exploring the effects of status hierarchies; see Sapolsky 2005). How might richer sociological, anthropological, and cultural-theoretical accounts - which attend to the complex ways in which poverty is produced and sustained - transform those models?

Except where otherwise noted, this work is licensed under a Creative Commons Attribution 3.0 Unported License. To view a copy of this license, visit https://creativecommons.org/licenses/by/3.0/ 